\title{
Anestesia epidural com associação medetomidina e lidocaína, em gatos pré-medicados com acepromazina e midazolam
}

[Epidural anesthesia with lidocaine and association medetomidine in cats pre-medicated with acepromazine and midazolam]

D.A.S.D. Lima $^{1}$, A.P. Souza ${ }^{2 *}$, V.L. Santana ${ }^{1}$, A.L. Araújo ${ }^{1}$, W.C. Lima ${ }^{1}$, R.S. Mendes ${ }^{3}$, P.I. Nóbrega Neto ${ }^{2}$

${ }^{1}$ Pós-graduação em Medicina Veterinária - UFCG - Campus de Patos, PB

${ }^{2}$ Universidade Federal de Campina Grande - UFCG - Unidade Acadêmica de Medicina Veterinária Av. Universitária, s/n - Bairro Sta. Cecília 58708-110 - Patos, PB

${ }^{3}$ Universidade Federal de Campina Grande - Campus de Patos, PB

\begin{abstract}
RESUMO
Avaliaram-se os efeitos anestésicos promovidos pela associação medetomidina e lidocaína por via epidural, em gatos pré-tratados com acepromazina e midazolam. Foram utilizados 10 gatos adultos, machos e fêmeas, hígidos e com média de peso de 2,5 $\pm 0,6 \mathrm{~kg}$, distribuídos em dois grupos (GM e GL) de igual número $(\mathrm{n}=5)$. Administraram-se, como medicação pré-anestésica, acepromazina, $0,2 \mathrm{mg} / \mathrm{kg}$, e midazolam, 0,5mg/kg, via intramuscular, e 20 minutos depois, nos animais do GM, por via epidural, lidocaína, $4,4 \mathrm{mg} / \mathrm{kg}$, associada à medetomidina, $0,02 \mathrm{mg} / \mathrm{kg}$. Os gatos do GL receberam lidocaína, $4,4 \mathrm{mg} / \mathrm{kg}$, associada à solução de $\mathrm{NaCl}$ a $0,9 \%$. As avaliações ocorreram antes da pré-anestesia (MPA), 20 minutos após a MPA e antes da anestesia epidural, e aos 10, 20, 30 e 40 minutos após a anestesia epidural, respectivamente, T-20, T0, T10, T20, T30 e T40. Foram avaliados: frequência cardíaca (FC) e respiratória (FR), temperatura do corpo, saturação de oxiemoglobina, analgesia, miorrelaxamento e período de recuperação. No GM, a FC diminuiu em T20, T30 e T40 em relação ao T-20 e T10 e foi mais baixa que a FC do GL em T20, T30 e T40, respectivamente, 86, 91 e 88 bat/min e 194, 205 e 177 bat/min. A FR variou entre o T-20 e os outros momentos de avaliação nos animais do GL. Nas variáveis eletrocardiográficas, houve diferenças entre T20, T30 e T40 e T-20 e T0, valores de 235, 238 e 240ms e 156 e 161ms, respectivamente, somente no GM. Este grupo diferiu do GL nas avaliações em T20, T30 e T40, valores de 147, 132 e 150ms para os gatos do GL. Oitenta por cento dos gatos tiveram analgesia intensa, e em todos os animais ocorreu relaxamento da mandíbula e da língua. O tempo de recuperação foi de 40 e $15 \mathrm{~min}$ no GM e no GL, respectivamente. Concluiu-se que a associação lidocaína com medetomidina promoveu plano anestésico estável com grau de anestesia e recuperação anestésica de boa qualidade.
\end{abstract}

Palavras-chave: $\alpha_{2}$-agonistas, anestesia, eletrocardiograma

\begin{abstract}
The anesthetic effects due to the association of medetomidine and epidural lidocaine in cats pretreated with acepromazine and midazolam were evaluated. Ten adult cats were used, male and female, healthy and weighing $2.5 \pm 0.6 \mathrm{~kg}$. They were divided into two groups (GM and GL) of equal numbers ( $n=5)$. Premedication with acepromazine, $0.2 \mathrm{mg} / \mathrm{kg}$, and midazolam, $0.5 \mathrm{mg} / \mathrm{kg}$, intramuscular was administered. Twenty minutes later, GM animals were given epidural lidocaine, $4.4 \mathrm{mg} / \mathrm{kg}$, associated with medetomidine, $0.02 \mathrm{mg} / \mathrm{kg}$. GL cats received lidocaine, $4.4 \mathrm{mg} / \mathrm{kg}$, associated with $\mathrm{NaCl} 0.9 \%$. Assessments occurred before the pre-anesthesia (MPA), 20 minutes after premedication and before the epidural block, and 10, 20, 30 and 40 minutes after epidural anesthesia, respectively, T-20, T0, T10, T20,
\end{abstract}

Recebido em 12 de março de 2010

Aceito em 21 de março de 2011

*Autor para correspondência (corresponding author)

E-mail: almir@cstr.ufcg.edu.br 
T30 and T40. Heart rate, respiratory rate, body temperature, oxyhemoglobin saturation, analgesia, muscle relaxation and recovery period were evaluated. In GM cats the heart rate decreased at T20, T30 and T40 as compared to T-20 and T10 and was lower than the heart rate in the GL cats at T20, T30 and T40, values being, respectively, 86, 91 and 88 beats/min and 194, 205 and 177 beats/min. The respiratory rate ranged from T-20 and the other time points in GL animals. Concerning electrocardiographic variables, in GM cats significant differences between T20, T30 and T40 and T0 and T-20, were observed, values being 235, 238 and 156 and 161ms and 240ms, respectively. GM animals differed from GL in T20, T30 and T40, values being 147, 132 and 150ms for GL cats. Eighty percent of the cats had severe pain and in all animals there was a relaxation of the jaw and tongue. The recovery time was 40 and 15 min at GM and GL, respectively. It was concluded that the association promoted lidocaine with medetomidine anesthesia with a stable level of anesthesia and anesthetic recovery of good quality.

Keywords: $\alpha_{2}$-agonist, anesthesia, electrocardiography

\section{INTRODUÇÃO}

A anestesia epidural tem destaque no controle da dor abdominal e de membros pélvicos tendo como principais vantagens o baixo custo, a eficiência e a segurança, além do fato de mínimos distúrbios bioquímicos e fisiológicos. Dentre os fármacos empregados para a promoção de anestesia epidural, podem-se citar a lidocaína, a bupivacaína, a cetamina e fármacos $\alpha_{2}$ agonistas (Jones, 2001). Tais substâncias podem ser usadas de forma isolada ou em associação, a fim de minimizar os efeitos indesejáveis decorrentes de seu uso isolado, bem como potencializar os efeitos bloqueadores motores e sensitivos, determinando, assim, maior conforto aos animais durante as intervenções cirúrgicas efetuadas.

A lidocaína é o anestésico local de maior aplicação no bloqueio epidural em cães e gatos (Otero, 2005; Skarda e Tranquilli, 2007). Promove o bloqueio da condução nervosa de forma reversível, interagindo com os canais de sódio na parte interna da membrana celular (Cortopassi et al., 2002a). Tem ação rápida e intensa, alta lipossolubilidade e duração de ação moderada (40 a 60 minutos), podendo chegar até 120 minutos pela associação com vasoconstritores. A administração por via epidural em gatos de um volume de $0,22 \mathrm{~mL} / \mathrm{kg}$ de lidocaína a $2 \%$ bloqueia a condução nervosa desde L1 em direção caudal (Hall et al., 2001), e com $0,31 \mathrm{~mL} / \mathrm{kg}$ consegue-se bloquear de T12 em diante na direção caudal (Otero, 2005).

A medetomidina é uma mistura racêmica de dois enantiômeros, a dexmedetomidina e a levomedetomidina, sendo esta última geralmente considerada farmacologicamente inativa, enquanto a primeira está ativa (Savola et al., 1991). É um potente $\alpha_{2}$-agonista que estimula não só os receptores $\alpha_{2}$ no sistema nervoso central e retém norepinefrina dentro das vesículas pré-sinápticas, produzindo sedação e analgesia dose-dependente, como também os receptores centrais e periféricos, causando bradicardia acentuada e diminuição do débito cardíaco (Cullen, 1996). O relaxamento muscular promovido decorre da inibição da transmissão interneuronal na medula espinhal (Otero, 2005). Segundo Jones (2001), a administração de medetomidina na dose de $15 \mu \mathrm{g} / \mathrm{kg}$ pela via epidural confere analgesia em cães e gatos por quatro a oito horas, estando a manifestação de efeitos colaterais por esta via relacionadas à incursão da substância na circulação sistêmica (Otero, 2005).

Alterações relativas à eletrocardiografia, obtidas em cães submetidos à medetomidina via epidural, foram investigadas por Vesal et al. (1996), que demonstraram retardo na transmissão (ou bloqueio) do impulso elétrico por meio do nodo atrioventricular (bloqueio atrioventricular de segundo grau) associado com arritmia sinusal por breve período de 20 minutos após a administração do fármaco.

A medetomidina em associação com a lidocaína é útil por promover significativa analgesia, proporcionando a realização de pequenos procedimentos cirúrgicos em cães (Sarkiala, 1989). Entretanto, não foram encontradas na literatura consultada informações referentes à administração da associação medetomidina e lidocaína pela via epidural em gatos.

Assim, este trabalho teve o objetivo de avaliar os efeitos anestésicos promovidos pela associação 
medetomidina e lidocaína por via epidural, em gatos pré-tratados com acepromazina e midazolam, bem como a manifestação de possíveis alterações eletrocardiográficas e clínicas.

\section{MATERIAL E MÉTODOS}

O estudo foi aprovado pelo Comitê de Ética e Pesquisa do Centro de Saúde e Tecnologia Rural da Universidade Federal de Campina Grande, Campus de Patos - PB, sob o protocolo de $n^{\circ}$ 122/2009.

Foram utilizados 10 gatos adultos, dois machos e oito fêmeas, com média de peso de $2,5 \pm 0,6 \mathrm{~kg}$, sem raça definida, considerados clinicamente sadios após exame físico geral, os quais foram mantidos em gatis individuais em Hospital Veterinário Escola. Os animais, distribuídos aleatoriamente em dois grupos (GM e GL), de igual número $(\mathrm{n}=5)$, foram submetidos, previamente, a jejum alimentar e hídrico de 12 e três horas, respectivamente. Em seguida, foi realizada a cateterização da veia cefálica esquerda ou direita para administração de solução de $\mathrm{NaCl}$ a $0,9 \%$, no volume de $10 \mathrm{~mL} / \mathrm{kg} / \mathrm{h}$ para a manutenção da hidratação do animal. Ato contínuo, foi administrado como medicação pré-anestésica (MPA) acepromazina (Acepran 1\% - Lab. Univet S.A. Indústria Veterinária - São Paulo, Brasil) - 0,2mg/kg - e midazolam (Dormonid 5mg/mL - Lab. Cristália Produtos Químicos e Farmacêuticos Ltda. Itapira, Brasil) - 0,5mg/kg - juntos, na mesma seringa, pela via intramuscular (IM). Decorridos 20 minutos, foi administrada aos animais do GM, por via epidural, associação de lidocaína (Cloridrato de lidocaína 2\% sem vasoconstritor Lab. Hipolabor - Sabará, Brasil) - 4,4mg/kg com medetomidina (Domitor - Lab. Pfizer Porto Salvo, Portugal) - 0,02mg/kg -, enquanto nos do GL foi aplicada lidocaína na mesma dose e via do GM associado à solução de $\mathrm{NaCl}$ a $0,9 \%$. O volume total da solução administrada foi ajustado para $0,30 \mathrm{~mL} / \mathrm{kg}$ em ambos os grupos conforme descrito por Lee et al. (2004).

Em ambos os grupos, efetuou-se registro do eletrocardiograma (ECG) em derivação DII, com emprego de eletrocardiógrafo computadorizado (TEB - mod. ECGPC software versão 1.10 - São Paulo, Brasil), pelo qual foram observados os valores referentes à duração e amplitude da onda P (Pms e PmV), duração do complexo QRS (QRSms), amplitude da onda R (RmV) e os intervalos em milissegundos ( $\mathrm{ms}$ ) entre as ondas $\mathrm{P}$ e R (PR), Q e T (QT) e entre duas ondas R subsequentes (RR). Também foi observada a presença, ou não, de figuras eletrocardiográficas anormais.

Foram mensuradas, ainda, frequência cardíaca (FC), calculada por meio do intervalo R-R obtido no eletrocardiógrafo computadorizado (bat $/ \mathrm{min}$ ); frequência respiratória (FR) (mov/min); temperatura retal (TR) $\left({ }^{\circ} \mathrm{C}\right)$ e saturação de oxiemoglobina $\left(\mathrm{SpO}_{2}\right)$, obtida em monitor multiparamétrico (INMAX COLOR - Instramed Indústria Médico Hospitalar Ltda. - Porto Alegre, Brasil), sendo o emissor/sensor adaptado em região corpórea dotada de grau de transparência compatível com a sensibilidade do dispositivo (lábios, orelhas, prepúcio, mamas) e registrada em \%.

O grau de relaxamento muscular foi avaliado a cada 10 minutos após a anestesia epidural, sendo determinado por meio do relaxamento da língua e da mandíbula e pelo reflexo interdigital nos membros torácicos. Para isso, utilizou-se uma escala de escores predeterminados: $1=$ discreto, $2=$ moderado e $3=$ intenso. A analgesia foi determinada segundo a resposta apresentada aos estímulos dolorosos decorrentes do pinçamento com pinça de Kocher $(15 \mathrm{~cm})$, até o primeiro dente da cremalheira, em áreas previamente determinadas (base do pescoço, base da cauda e espaço interdigital do membro torácico e pélvico) após a avaliação das outras variáveis, utilizando-se os escores 1= discreta, 2= moderada e $3=$ intensa, quando presente.

Os momentos estabelecidos para o registro das variáveis foram: T-20 - antes da administração da medicação pré-anestésica -, T0 - 20 minutos após a administração da MPA e antes da anestesia epidural -, e de 10 em 10 minutos após a anestesia epidural durante 40 minutos (T10, T20, T30 e T40). Avaliaram-se, ainda, o tempo de recuperação anestésica (minutos), compreendido entre o término do período hábil anestésico e o início da deambulação espontânea, e a manifestação de reações indesejáveis como vocalização, tremores, comportamento de fuga e/ou agitação, vômito e defecação. 
Foi utilizada a análise de variância (ANOVA), seguida do teste Tukey ( $\mathrm{P}<0,05)$, usando-se o programa GraphPad InStat (GraphPad Software version 3.00 for Windows, San Diego, California, USA). Para avaliação dos dados referentes ao período de recuperação, foi utilizada a ANOVA, seguida do teste $\mathrm{t}(\mathrm{P}<0,05)$.

\section{RESULTADOS E DISCUSSÃO}

A FC foi significativamente mais baixa no GM em T10, T20, T30 e T40 (Tab. 1). Na comparação dos momentos em cada grupo, observou-se que no GM as médias de T20, T30 e T40 foram mais baixas que as dos demais momentos, enquanto no GL não houve diferença entre momentos. De forma geral, fenotiazínicos e benzodiazepínicos não acarretam alterações cardiovasculares significativas (Natalini, 2007), estando a redução da FC observada no GM após a administração da anestesia epidural relacionada ao mecanismo de ação do $\alpha_{2}$-agonista utilizado, fármaco com reconhecida ação bradicardizante decorrente do bloqueio dos receptores noradrenérgicos pré-sinápticos centrais e periféricos, em que o tônus vagal aumenta pela redução da atividade simpática e promove bradicardia e hipotensão (Ansah, 2004; Natalini, 2007). No GL, nenhuma alteração foi observada na FC, haja vista que alterações no ritmo cardíaco e depressão do miocárdio são transtornos registrados quando ocorre toxicidade com os anestésicos locais (Otero, 2005; Skarda e Tranquilli, 2007).

A FR não teve variação significava entre os grupos (Tab. 1). Entre os momentos no GL, as médias em T0, T10, T20 e T30 foram significativamente menores que as demais, enquanto no GM não houve diferença significativa $(\mathrm{P}>0,05)$ entre os momentos. A redução acentuada da FR entre T-20 e T0, em ambos os grupos, é atribuída à MPA, visto que a acepromazina diminui a sensibilidade dos quimiorreceptores ao dióxido de carbono, podendo diminuir a FR (Cortopassi et al., 2002b). O midazolam, por sua vez, apesar de não promover alterações importantes nos parâmetros fisiológicos, potencializa os efeitos dos fenotiazínicos quando empregados concomitantemente (Natalini, 2007). Apesar de relatos existentes na literatura de que fármacos $\alpha_{2}$-agonistas promovem depressão da FR, verifica-se que esta é de caráter dose-dependente, sem alterações significativas na ventilação do paciente (Kuusela et al., 2001 ab; Otero, 2005; Gómez-Villamandos et al., 2006). Dessa forma, a ausência de efeitos depressores neste estudo poderia estar relacionada à dose utilizada, determinada em estudo prévio, e baseada em descrições de literatura (Duke et al., 1994; Ansah et al., 1998), bem como à via de administração epidural -, que determinaria menores níveis plasmáticos do fármaco. No tocante ao uso da lidocaína, Otero (2005) afirmou que apenas mediante o uso de grandes volumes de anestésicos locais pela via epidural é que se pode comprometer a função dos músculos responsáveis pela mecânica ventilatória por tempos prolongados, fato que não aconteceu neste estudo.

A TR não diferiu $(\mathrm{P}>0,05)$ entre os momentos e entre os grupos. A TR, logo após a MPA e ao longo dos momentos, alcançou valores abaixo dos limites considerados fisiológicos para a espécie (Futema, 2002), em ambos os grupos. Isso pode ser atribuído ao uso da acepromazina, que promove hipotermia pela depleção das reservas de dopamina no centro termorregulador (hipotálamo) e pela sua ação vasodilatadora periférica (Arena et al., 2009), permitindo maior perda de calor corporal por condução. Os $\alpha_{2}$ agonistas, por sua vez, induzem prolongada depressão na termorregulação, deprimindo receptores $\alpha_{2}$ noradrenérgicos hipotalâmicos (Ansah et al., 1998; Granholm et al., 2006), justificando a intensificação da hipotermia observada no GM, também registrada por Souza (2006), a qual permaneceu após o encerramento do período experimental. Adicionalmente, sabese que os gatos são mais propensos à hipotermia pela ação dos $\alpha_{2}$-agonistas do que os cães (Doherty, 1988).

A $\mathrm{SPO}_{2}$ não diferiu $(\mathrm{P}>0,05)$ entre os momentos e entre os grupos (Tab. 1). A média de valores esteve, desde o primeiro momento, próxima ao esperado para o paciente anestesiado, ou seja, 95\% de saturação (Haskins, 2007), o que está de acordo com o estudo de Souza (2006), em gatas. Tais valores são condizentes com o declínio observado na frequência respiratória. Deve ser ressaltado, porém, que os animais deste experimento estiveram durante todo o período experimental inalando ar atmosférico, o que dificultou a manutenção da $\mathrm{SpO}_{2}$ dentro dos limites ideais. 
Tabela 1. Médias $(\overline{\mathrm{X}})$ e desvios-padrão (s) das frequências cardíaca (FC) e respiratória (FR), da temperatura retal (TR) e da saturação de oxiemoglobina $\left(\mathrm{SpO}_{2}\right)$ obtidas em gatos anestesiados com a associação medetomidina e lidocaína (GM) ou lidocaína (GL), pela via epidural, ao longo dos momentos

\begin{tabular}{|c|c|c|c|c|c|c|c|c|}
\hline \multirow{2}{*}{ Variável } & \multirow{2}{*}{ Grupo } & & \multicolumn{6}{|c|}{ Momento } \\
\hline & & & $\mathrm{T}-20$ & T0 & T10 & T20 & T30 & T40 \\
\hline \multirow{4}{*}{$\begin{array}{l}\text { FC } \\
\text { (bat/min) }\end{array}$} & \multirow{2}{*}{ GM } & $\bar{x}$ & 174ab & $197 a$ & 116Babc & $86 \mathrm{Bc}$ & 91Bc & $88 \mathrm{Bc}$ \\
\hline & & $\mathrm{s}$ & 25,27 & 38,42 & 18,02 & 15,68 & 14,27 & 16,98 \\
\hline & \multirow{2}{*}{ GL } & $\bar{x}$ & 190 & 176 & $183 \mathrm{~A}$ & $194 \mathrm{~A}$ & $205 \mathrm{~A}$ & $177 \mathrm{~A}$ \\
\hline & & $\mathrm{s}$ & 31,04 & 21,67 & 33,08 & 37,64 & 46,99 & 39,55 \\
\hline \multirow{4}{*}{$\begin{array}{l}\text { FR } \\
\text { (mov/min) }\end{array}$} & \multirow{2}{*}{ GM } & $\bar{x}$ & 61 & 39 & 43 & 42 & 38 & 38 \\
\hline & & $\mathrm{s}$ & 17,30 & 12,13 & 12,13 & 4,56 & 6,07 & 2,19 \\
\hline & \multirow{2}{*}{ GL } & $\bar{x}$ & $63 a$ & $34 \mathrm{~b}$ & $34 \mathrm{~b}$ & $35 b$ & $32 b$ & $39 a$ \\
\hline & & $\mathrm{s}$ & 15,07 & 6,69 & 7,27 & 14,39 & 11,31 & 19,70 \\
\hline \multirow{4}{*}{$\begin{array}{l}\text { TR } \\
\left({ }^{\circ} \mathrm{C}\right)\end{array}$} & \multirow{2}{*}{ GM } & $\bar{x}$ & 38,4 & 38,1 & 37,5 & 37,1 & 37,0 & 36,8 \\
\hline & & s & 0,68 & 0,89 & 0,93 & 1,20 & 1,23 & 1,08 \\
\hline & \multirow{2}{*}{ GL } & $\bar{x}$ & 38,2 & 37,6 & 37,2 & 37,1 & 37,0 & 36,8 \\
\hline & & $\mathrm{s}$ & 0,54 & 0,44 & 0,96 & 1,06 & 1,05 & 1,0 \\
\hline \multirow{4}{*}{$\begin{array}{l}\mathrm{SpO}_{2} \\
\text { (\%) }\end{array}$} & \multirow{2}{*}{ GM } & $\bar{x}$ & 92 & 93 & 94 & 94 & 96 & 94 \\
\hline & & $\mathrm{s}$ & 5,68 & 5,94 & 2,95 & 2,70 & 1,95 & 1,09 \\
\hline & \multirow{2}{*}{ GL } & $\bar{x}$ & 89 & 89 & 94 & 91 & 94 & 91 \\
\hline & & $\mathrm{s}$ & 6,06 & 8,84 & 3,65 & 6,42 & 3,11 & 4,78 \\
\hline
\end{tabular}

Médias seguidas por letras diferentes, minúsculas na linha e maiúsculas na coluna, representam diferenças entre os valores $(\mathrm{P}<0,05)$.

Não foram verificadas manifestações de figuras eletrocardiográficas anormais. Os valores referentes à onda $\mathrm{P}(\mathrm{ms}$ e $\mathrm{mV})$ mostraram que não houve diferenças entre os grupos e ao longo dos momentos ( $\mathrm{P}>0,05)$. A variação da média de Pms obtida nos momentos, em cada grupo, foi pequena. Elevações da média acima daquela preconizada pela literatura (Camacho e Mucha, 2004) indicam alterações no tempo e na intensidade elétrica necessários para a promoção da despolarização atrial (Tilley, 1992), que neste estudo, não foi influenciada pelos protocolos anestésicos empregados.

Com relação ao tempo de condução elétrica no nodo atrioventricular (intervalo PR), não houve diferença entre os grupos e entre os momentos $(P>0,05)$. Os valores encontrados estão dentro da faixa de normalidade citada para a espécie (Tilley, 1992), não sendo verificada, dessa forma, qualquer interferência dos fármacos na condutibilidade elétrica no nodo citado e, consequentemente, na manifestação de bloqueio atrioventricular (BAV) de primeiro e segundo graus.

Pode-se afirmar que o tempo de despolarização ventricular manteve-se estável ao longo de todo o período de avaliação anestésica em ambos os grupos, visto que não houve diferenças dignas de nota ao longo dos momentos e entre grupos $(\mathrm{P}>0,05)$ nos valores referentes à duração do complexo QRS e à amplitude da onda $\mathrm{R}(\mathrm{mV})$.

O intervalo QT é utilizado para monitorar efeitos de fármacos e eletrólitos sobre a dinâmica cardíaca. Representa a sístole ventricular e é inversamente proporcional à FC (Tilley, 1992). Dessa forma, constatou-se que as maiores médias ocorreram no GM em T10, T20, T30 e T40 (Tab. 2), manifestação esta que pode ser justificada pelo efeito simpatolítico do agente $\alpha_{2}$ adrenérgico, o qual promove significativa redução da frequência cardíaca, como discutido 
anteriormente. O mesmo fato não se pode verificar para os animais do GL, pois não ocorreu interferência na frequência cardíaca. Houve diferença significativa entre os grupos em T20, T30 e T40 ( $\mathrm{P}<0,05)$, em que os valores de GM foram maiores em relação aos do GL, em função da resposta simpatolítica já comentada.
O intervalo RR representa, em síntese, as alterações referentes à frequência cardíaca (Tilley, 1992). Dessa forma, as variações verificadas ao longo do período experimental e entre os grupos são decorrentes dos efeitos dos fármacos sobre a frequência cardíaca, conforme já discutido.

Tabela 2. Médias ( $\overline{\mathrm{X}}$ ) e desvios-padrão (s) de variáveis eletrocardiográficas obtidas em gatos anestesiados com a associação medetomidina e lidocaína (GM) ou lidocaína (GL), pela via epidural, ao longo dos momentos

\begin{tabular}{|c|c|c|c|c|c|c|c|c|}
\hline \multirow{2}{*}{ Variável } & \multirow{2}{*}{ Grupo } & & \multicolumn{6}{|c|}{ Momento } \\
\hline & & & $\mathrm{T}-20$ & T0 & T10 & T20 & T30 & T40 \\
\hline \multirow{4}{*}{$\mathrm{P}(\mathrm{ms})$} & \multirow{2}{*}{ GM } & $\overline{\mathrm{x}}$ & 41,8 & 37,4 & 46,0 & 44,4 & 46,8 & 43,2 \\
\hline & & $\hat{\mathrm{S}}$ & 6,14 & 2,88 & 9,19 & 8,65 & 6,42 & 8,17 \\
\hline & \multirow{2}{*}{ GL } & $\overline{\mathrm{x}}$ & 40,0 & 42,6 & 44,8 & 46,0 & 46,0 & 41,5 \\
\hline & & $\mathrm{s}$ & 6,63 & 7,63 & 5,02 & 5,87 & 6,24 & 9,47 \\
\hline \multirow{4}{*}{$\mathrm{P}(\mathrm{mV})$} & \multirow{2}{*}{ GM } & $\overline{\mathrm{x}}$ & 0,08 & 0,10 & 0,08 & 0,08 & 0,07 & 0,08 \\
\hline & & $\mathrm{s}$ & 0,01 & 0,03 & 0,01 & 0,02 & 0,01 & 0,02 \\
\hline & \multirow{2}{*}{ GL } & $\overline{\mathrm{x}}$ & 0,07 & 0,07 & 0,08 & 0,08 & 0,10 & 0,09 \\
\hline & & $\mathrm{s}$ & 0,01 & 0,02 & 0,02 & 0,02 & 0,03 & 0,01 \\
\hline \multirow{4}{*}{ QRS (ms) } & \multirow{2}{*}{ GM } & $\overline{\mathrm{x}}$ & 36,4 & 32,8 & 39,6 & 41,0 & 41,2 & 38,0 \\
\hline & & $\mathrm{s}$ & 11,74 & 8,32 & 10,50 & 15,17 & 19,68 & 14,30 \\
\hline & \multirow{2}{*}{ GL } & $\overline{\mathrm{x}}$ & 47,8 & 38,0 & 42,2 & 34,6 & 49,8 & 43,2 \\
\hline & & $\mathrm{s}$ & 25,31 & 8,89 & 8,67 & 8,08 & 25,13 & 2,87 \\
\hline \multirow{4}{*}{$\mathrm{R}(\mathrm{mV})$} & \multirow{2}{*}{$\mathrm{GM}$} & $\overline{\mathrm{x}}$ & 0,23 & 0,21 & 0,34 & 0,32 & 0,32 & 0,23 \\
\hline & & $\mathrm{s}$ & 0,08 & 0,13 & 0,15 & 0,16 & 0,15 & 0,18 \\
\hline & \multirow{2}{*}{ GL } & $\overline{\mathrm{x}}$ & 0,17 & 0,17 & 0,17 & 0,15 & 0,19 & 0,17 \\
\hline & & $\hat{\mathrm{S}}$ & 0,06 & 0,07 & 0,06 & 0,05 & 0,08 & 0,06 \\
\hline \multirow{4}{*}{ QT (ms) } & \multirow{2}{*}{ GM } & $\bar{x}$ & $155,8 \mathrm{c}$ & $161,4 \mathrm{bc}$ & $221,4 a b$ & $234,8 \mathrm{Aa}$ & $238,2 \mathrm{Aa}$ & $240,0 \mathrm{Aa}$ \\
\hline & & $\mathrm{s}$ & 23,34 & 25,27 & 36,86 & 48,17 & 45,91 & 50,89 \\
\hline & \multirow{2}{*}{ GL } & $\bar{x}$ & 159,8 & 171,8 & 164,4 & $147,4 \mathrm{~B}$ & $132,4 \mathrm{~B}$ & $150,0 \mathrm{~B}$ \\
\hline & & $\mathrm{s}$ & 19,41 & 23,60 & 26,36 & 24,15 & 40,52 & 26,70 \\
\hline \multirow{4}{*}{ PR (ms) } & \multirow{2}{*}{ GM } & $\overline{\mathrm{x}}$ & 96,8 & 67,4 & 80,6 & 77,4 & 81,4 & 82,6 \\
\hline & & $\mathrm{s}$ & 56,27 & 3,58 & 6,43 & 6,58 & 4,34 & 2,88 \\
\hline & \multirow{2}{*}{ GL } & $\overline{\mathrm{x}}$ & 74,8 & 78,2 & 77,4 & 82,6 & 78,0 & 79,0 \\
\hline & & $\mathrm{s}$ & 10,96 & 7,56 & 13,72 & 10,14 & 12,21 & 7,12 \\
\hline \multirow{4}{*}{ RR (ms) } & \multirow[t]{2}{*}{ GM } & $\overline{\mathrm{x}}$ & $698,8 b$ & $628,0 \mathrm{~b}$ & $1019,8 b$ & 1382,6Aa & $1360,8 \mathrm{Aa}$ & $\begin{array}{l}1394,6 \mathrm{~A} \\
\mathrm{a}\end{array}$ \\
\hline & & $\mathrm{s}$ & 101,65 & 117,77 & 229,95 & 198,58 & 281,57 & 311,25 \\
\hline & \multirow{2}{*}{ GL } & $\bar{x}$ & 642,8 & 688,6 & 671,8 & $640,6 \mathrm{~B}$ & $610,2 B$ & $699,0 \mathrm{~B}$ \\
\hline & & $\mathrm{s}$ & 105,80 & 90,72 & 118,23 & 144,24 & 150,41 & 176,82 \\
\hline
\end{tabular}

Médias seguidas por letras diferentes, minúsculas na linha e maiúsculas na coluna, representam diferenças entre os valores $(\mathrm{P}<0,05)$. 
Com relação ao grau de analgesia, no GM 80\% dos animais tiveram analgesia intensa e $20 \%$ moderada a discreta. No GL, a analgesia foi considerada discreta, com redução da sensibilidade apenas na região pélvica, que se estendeu até a quinta vértebra lombar (L5), com diminuição do grau de analgesia ao longo dos momentos, enquanto no GM esta se manteve persistente por até 80 minutos. Sabe-se que a medetomidina promove analgesia inibindo a liberação de noradrenalina por retroalimentação negativa e na região pós-sináptica em diversos locais do organismo (Bacchiega et al., 2008), aumentando significantemente o limiar de dor nos membros anteriores, conforme já registrado por Duke et al. (1994).

No GM, imediatamente após a anestesia epidural, foram observados aumento da salivação em $80 \%$ dos animais, ânsia de vômito em $60 \%$ e êmese em 20\%. A êmese é mediada pela ativação de receptores $\alpha_{2}$-adrenérgicos no sistema nervoso central e pela diminuição do tônus do esfíncter gastroesofágico (Thurmon et al., 1996). Duke et al. (1994) relataram vômito em $80 \%$ dos gatos, produzido pela medetomidina via epidural, seis minutos após a administração do fármaco. O baixo percentual de animais deste estudo com manifestação de vômitos pode estar relacionado à acepromazina empregada na MPA, a qual apresenta, entre outros efeitos, bloqueio dopaminérgico do centro do vômito no sistema nervoso central (Lemke, 2007).

Em todos os animais do GM, foi observado intenso relaxamento da mandíbula e da língua, sem retração desta, com reflexo interdigital nos membros torácicos deprimido (retirada lenta após estímulo), sinais que persistiram por até 80 minutos após a anestesia epidural.

O grau de relaxamento muscular central produzido pela medetomidina, dexmedetomidina e xilazina é considerado de boa qualidade (Vainio et al., 1989; Ansah et al., 1998) e está relacionado à inibição da transmissão intraneuronal dos impulsos no sistema nervoso central (Gross, 2003), sendo encontrado, também, por Scrollavezza et al. (2009) e Steagall et al. (2009). No GL, entretanto, nenhum animal apresentou relaxamento muscular satisfatório segundo o método de avaliação utilizado.
O tempo de recuperação (término da anestesia e

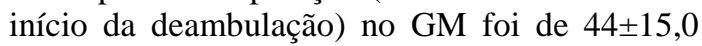
minutos, e no GL, de 15,4 \pm 7,5 minutos. Os efeitos sedativos da administração epidural de medetomidina no GM aumentaram o período de monitoração da recuperação anestésica. Durante esse período, foram observados no GL movimentos involuntários dos membros com aumento da resposta motora ao estímulo do avaliador, ataxia discreta, características de excitação e comportamento de fuga. No GM, os animais apresentaram recuperação suave e sedação de boa qualidade, sem incidência de delírios e/ou tremores musculares, benefícios promovidos pelo efeito sedativo da medetomidina.

\section{CONCLUSÕES}

A associação medetomidina e lidocaína por via epidural, em gatos pré-medicados com acepromazina e midazolam, reduz a frequência cardíaca sem promover retardo tanto na condução elétrica atrial quanto na ventricular. Contudo, promove plano anestésico mais estável, com melhor grau de anestesia e recuperação anestésica de melhor qualidade.

\section{AGRADECIMENTOS}

Ao Conselho Nacional de Desenvolvimento Científico e Tecnológico - CNPq, pela concessão da bolsa de estudo.

\section{REFERÊNCIAS BIBLIOGRÁFICAS}

ANSAH, O.B.; RAEKALLIO, M.; VAINIO, O. Comparison of three doses of dexmedetomidine with medetomidine in cats following intramuscular administration. J. Vet. Pharm. Ther., v.21, p.380-387, 1998.

ANSAH, O.B. Use of the alpha-2-adrenoceptor agonists medetomidine and dexmedetomidine in the sedation and analgesia of domestic cats. 2004. 93f. Academic Dissertation - University of Helsinki, Faculty of Veterinary Medicine, Department of Clinical Veterinary Sciences, Clinical Pharmacology - Anaesthesia, Finland. Disponível em: $<$ http://ethesis.helsinki.fi/julkaisut/ela/kliin/vk/an sah/>. Acessado em: 29 nov. 2009. 
ARENA, G.; BOTELHO, A.; EVARISTO, B. et al. Fenotiazínicos: usos, efeitos e toxicidade em animais de grande e pequeno porte. Rev. Cient. Elet. Med. Vet., v.7, 2009.

BACCHIEGA, T.S.; SIMAS, R.C.; PINTO, E.A.T. Dexmedetomidina, um novo medicamento na anestesiologia veterinária. Rev. Cient. Elet. Med. Vet., v.6, 2008. Disponível em: www.revista.inf.br/veterinaria10/revisao/edic-vin10-RL28.pdf>. Acessado em: 29 nov. 2009.

CAMACHO, A.A.; MUCHA, C.J. Semiologia do sistema circulatório de cães e gatos. In: FEITOSA, F.L.F. Semiologia Veterinária - A arte do diagnóstico. São Paulo: Roca, 2004. p.282-311.

CORTOPASSI, S.R.G.; FANTONI, D.T.; BERNARDI, M.M. Anestesia local In: SPINOSA, H.S.; GÓRNIAK, S.L.; BERNARDI, M.M. Farmacologia aplicada à medicina veterinária. 3.ed. Rio de Janeiro: Guanabara Koogan, 2002a. p.129-136.

CORTOPASSI, S.R.G.; FANTONI, D.T. Medicação pré-anestésica. In: FANTONI, D.T.; CORTOPASSI, S.R.G. Anestesia em cães $e$ gatos. São Paulo: Roca, 2002b. p.151-158.

CULLEN, L.K. Medetomidine sedation in dogs and cats: a review of its pharmacology, antagonism and dose. Br. Vet. J., v.5, p.519-535, 1996.

DOHERTY, T.J. Phisiologic effects of alpha2adrenergic receptors. J. Am. Vet. Med. Assoc., v.192, p.1612-1614, 1988.

DUKE, T.; KOMULAINEN, A-M.; REMEDIOS, A.M. et al. The analgesic effects of administering fentanyl or medetomidine in the lumbosacral epidural space of cats. Vet. Surg., v.23, p.143-148, 1994.

FUTEMA, F. Avaliação pré-anestésica. In: FANTONI, D. T.; CORTOPASSI, S. R. G. Anestesia em cães e gatos. São Paulo: Roca, 2002. p.59-63.

GÓMEZ-VILLAMANDOS, L.J.; PALACIOS, C.; BENÍTEZ, A. et al. Dexmedetomidine or medetomidina premedication before propofoldesflurane anaesthesia in dogs. J. Vet. Pharm. Therap., v.29, p.157-163, 2006.
GRANHOLM, M.; McKUSICK, B.; WESTERHOLM, F.C. et al. Evaluation of the clinical efficacy and safety of dexmedetomidine or medetomidine in cats and their reversal with atipamezole. Vet. Anaesth. Analg., v.33, p.214223, 2006.

GROSS, M.E. Tranquilizantes, agonistas $\alpha_{2}{ }^{-}$ adrenérgicos e agentes relacionados. In: ADAMS, H.R. Farmacologia e terapêutica em veterinária. 8.ed. Rio de Janeiro: Guanabara Koogan, 2003. p.249-283.

HALL, L.W.; CLARKE, K.W.; TRIM, C.M. Veterinary anaesthesia. 10.ed. Philadelphia: Saunders, 2001. p.225-461.

HASKINS, S.C. Monitoring anesthetized patients. In: TRANQUILLI, W.J.; THURMON, J.C.; GRIMM, K.A. Lumb and Jones' veterinary anaesthesia. Baltimore: Williams \& Wilkins, 2007. p. 533-560.

JONES, R.S. Epidural analgesia in the dog and cat. Vet. J., v.161, p.123-131, 2001.

KUUSELA, E.; RAEKALLIO, M.; VÄISÄNEN, $\mathrm{M}$. et al. Comparison of medetomidine and dexmedetomidine as premedicants in dogs undergoing propofol-isoflurane anesthesia. Am. J. Vet. Res., v.62, p.1073-1080, 2001a.

KUUSELA, E.; VAINIO, O.; KAISTENEN, A. et al. Sedative, analgesic, and cardiovascular effects of levomedetomidine alone and in combination with dexmedetomidine in dogs. Am. J. Vet. Res., v.62, p.616-621, 2001b.

LEE, I.; YAMAGISHI, N.; OBOSHI, K. et al. Distribution of new methylene blue injected into the lumbosacral epidural space in cats. Vet. Anaesth. Analg., v.31, p.190-194, 2004.

LEMKE, K.A. Anticholinergics and sedatives. In: TRANQUILLI, W.J.; THURMON, J.C.; GRIMM, K.A. Lumb and Jones' veterinary anaesthesia. Baltimore: Williams \& Wilkins, 2007. p. 203-240.

NATALINI, C.C. Teoria e técnicas em anestesiologia veterinária. Porto Alegre: Artmed, 2007. p. 43-64.

OTERO, P. Dor: avaliação e tratamento em pequenos animais. São Paulo: Interbook, 2005. Cap.14, p.192-211. 
SARKIALA, E. Suturation of wounds of extremities in dogs using medetomidine as sedative and analgesic drug. Acta Vet. Scand., v.85, suppl., p.175-177, 1989.

SAVOLA, J.M.; VIRTANEN, R. Central alpha2adrenoceptors are highly stereoselective for dexmedetomidine, the dextro enantiomer of medetomidine. Eur. J. Pharmacol., v.2, p.193199, 1991.

SCROLLAVEZZA, P.; TAMBELLA, A.M.; VULLO, C. et al. Evaluation of the muscular relaxant effect of dexmedetomidine or medetomidine in cats. Vet. Res. Commun., v.33, suppl. 1, p. S213-S215, 2009.

SKARDA, R.T.; TRANQUILLI, W.J. Local and regional anaesthetic and analgesic techiniques: cats. In: TRANQUILLI, W.J.; THURMON, J.C.; GRIMM, K.A. Lumb and Jones' veterinary anaesthesia. Baltimore: Williams \& Wilkins, 2007. p.595-604.

SOUZA, S.S. Efeitos da dexmedetomidina, por via epidural ou infusão contínua intravenosa, em gatas anestesiadas com propofol e isofluorano $e$ submetidas a ovariossalpingoisterectomia. 2006. 140f. Dissertação (Mestrado em Medicina Veterinária) - Faculdade de Medicina Veterinária e Zootecnia, Universidade de São Paulo, São Paulo, SP.
STEAGALL, P.V.; MILLETTE, V.; MANTOVANI, F.B. et al. Antinociceptive effects of epidural buprenorphine or medetomidine, or the combination, in conscious cats. J. Vet. Pharmacol. Ther., v.32, p.477-84, 2009.

TILLEY, L.P. Analysys of canine P-QRS-T deflections. In:__ Essential of canine and feline eletrocardiography. 3.ed. Philadelphia: Lea \& Febiger, 1992. p.59-99.

THURMON, J.C.; TRANQUILLI, W.J.; BENSON, G.J. Lumb and Jones' veterinary anaesthesia. 3.ed. Philadelphia: Lea \& Feabiger, 1996. Preanesthetics and anesthetic adjuncts: p.183-209.

VAINIO, O.; VÄHÄ-VAHE, T.; PALMU, L. Sedative and analgesic effect of medetomidine in the dogs. J. Vet. Pharmacol. Ther., v.12, p.225231, 1989.

VESAL, N.; CRIBB, P.H.; FRKETIC, M. Postoperative analgesic and cardiopulmonary effects in dogs of oxymorphone administered epidurally and intramuscularly, and medetomidine administered epidurally: a comparative clinical study. Vet. Surg., v.25, p.361-369, 1996. 